\title{
ANÁLISE DOS TRABALHOS QUE USARAM O MODELO LEVERS OF CONTROL DE SIMONS NA LITERATURA INTERNACIONAL NO PERÍODO DE 1995 A 2007
}

\section{ANALYSIS OF PAPERS ON SIMON'S LEVERS OF CONTROL MODEL ON INTERNATIONAL LITERATURE DURING THE PERIOD OF 1995 TO 2007}

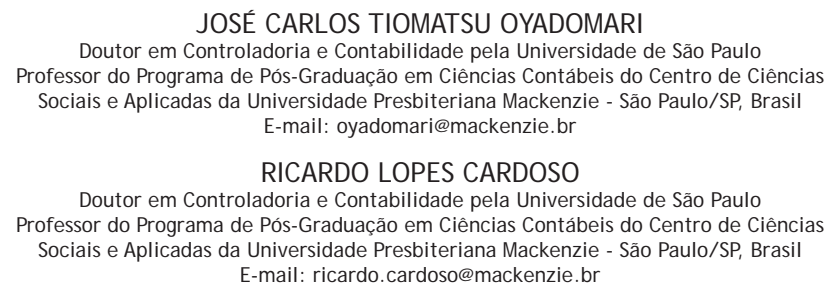

J OSÉ CARLOS TIOMATSU OYADOMARI

Doutor em Controladoria e Contabilidade pela Universidade de São Paulo Professor do Programa de Pós-Graduação em Ciências Contábeis do Centro de Ciências Sociais e Aplicadas da Universidade Presbiteriana Mackenzie - São Paulo/ SP, Brasil E-mail: oyadomari@mackenzie.br RICARDO LOPES CARDOSO

Doutor em Controladoria e Contabilidade pela Universidade de São Paulo Professor do Programa de Pós-Graduação em Ciências Contábeis do Centro de Ciências Sociais e Aplicadas da Universidade Presbiteriana Mackenzie - São Paulo/SP, Brasil E-mail: ricardo.cardoso@mackenzie.br

\author{
FÁBIO FREZATTI \\ Livre-Docente pela Universidade de São Paulo \\ Professor Titular da Faculdade de Economia, Administração e \\ Contabilidade da Universidade de São Paulo - São Paulo/ SP, Brasil \\ E-mail: frezatti@usp.br \\ ANDSON BRAGA DE AGUIAR \\ Doutor em Controladoria e Contabilidade pela Universidade de São Paulo \\ Professor e Pesquisador da Fucape Business School- Vitória/ ES \\ E-mail: andsonbraga@fucape.br
}

A dimensão Uso do Sistema de Controle Gerencial (SCG) proposta por Simons é referendada como inovadora por reforçar o papel do SCG como influenciador da estratégia e, portanto, com possibilidade de ser estudado em pesquisas que expliquem as diferenças de desempenho das empresas. Esta importância pode ser verificada pelos trabalhos que utilizaram parcial ou integralmente o framework Levers of Control no período de 1995 a 2007, publicados nos principais periódicos internacionais de Contabilidade Gerencial e Estratégia, sem que seja encontrada correspondência na literatura nacional. Este artigo teve como objetivo identificar quais contribuições os estudos publicados na literatura internacional sobre o modelo Levers of Control podem trazer para a pesquisa em Contabilidade Gerencial no Brasil. O trabalho tem como principais contribuições propiciar uma análise aprofundada do modelo teórico e consolidar os resultados empíricos acerca do construto. Do ponto de vista metodológico, o trabalho se caracteriza como uma análise bibliográfica complementada por uma análise crítica dos resultados das pesquisas. Os resultados desta análise evidenciaram que grande parte do modelo foi validada empiricamente, embora algumas pesquisas trouxeram resultados divergentes. Outra constatação é que as pesquisas não tiveram continuidade, de onde se conclui que o modelo tem grande potencial para ser validado em pesquisas brasileiras. Recomenda-se o estudo do modelo em conjunto com construtos da Estratégia e Comportamento Organizacional, assumindo que ele possa ser apenas parte de um framework mais amplo.

Palavras-chave: Simons, Levers of Control, Uso Interativo.

\section{ABSTRACT}

The dimension of Management control system (MCS) use proposed by Simons is considered innovative because it strengthens the MCS role as a strategy inducer, so it has the possibility to be studied in researches that explain the companies different performances. This importance can be verified in the papers that used, partially or fully, the framework Levers of Control during the period of 1995 to 2007, which were published in the main international magazines of Management Accountancy and Strategy, lacking any correspondence in the national literature. This article had as its objective to identify which contributions the studies published in the international literature about the model Levers of Control could bring to the research in Management Accountancy in Brazil. The paper has as its main contributions to provide a deep analysis of the theoretical model and to consolidate the empirical results about the construct. According to the methodological point of view, the paper is taken as a bibliographic analisys added by a critical analysis of the results of researches. The results of this analysis showed that most of the model was validated empirically, although some researches brought up different results. Another finding is that the researches had no continuity and because of that it is concluded that the model has great potential to be validated in Brazilian researches. It is recommended the study of the model in association with constructs of Strategy and Organizational Behavior, assuming that it can be only a part of a greater framework.

Key-words: Simons, Levers of Control, Interactive use. 


\section{INTRODUÇÃO}

Estudos desenvolvidos sob a perspectiva da Teoria Institucional em sua vertente, Nova Sociologia Institucional, tem mostrado que os artefatos de contabilidade gerencial utilizados pelas grandes empresas são os classificados como artefatos tradicionais (SOUZA et al., 2003; FREZATTI, 2006), com as empresas motivadas ao isomorfismo (DIMAGGIO e POWELL, 1983). Em uma visão pragmática, a Contabilidade Gerencial busca utilizar os artefatos para melhorar o desempenho das empresas e obter melhores desempenhos. É a tônica de executivos e estudiosos da estratégia. Portanto, se os artefatos existentes são muito semel hantes entre as empresas, logo a sua adoção não poderia explicar os diferentes desempenhos das empresas, o que enseja a necessidade de estudar uma nova dimensão, o Uso do Sistema de Controle Gerencial (SCG).

Simons $(1995,2000)$ é pioneiro em desenvolver uma tipologia para o uso do SCG, classificando-o em Uso Diagnóstico e Uso Interativo. Embora a dimensão Uso do Sistema de Controle Gerencial seja pouco estudada no Brasil, internacionalmente existem trabalhos que exploram esse construto. Ferreira e Otley (2006) referendam a tipologia proposta por Simons relativa ao uso do SCG, afirmando que esse é um modelo mais adequado que o Estilo de Avaliação de Desempenho proposto por Hopwood (1972), já que abrange outras dimensões, além do orçamento. Jönsson (2009) reconhece o trabalho de Simons como a abordagem do uso de diferentes partes do sistema de controle gerencial para diferentes propósitos. Chapman et al. (2009) também se referem à validade do modelo citando o trabalho empírico de Widener (2007) aplicado ao Levers of Control. Bisbe e Malagueño (2009) definem Levers of Control como uma literatura emergente em Contabilidade Gerencial.

A relevância do trabalho de Simons está também na abordagem que caracterizou o Sistema de Controle Gerencial como influenciador da estratégia, e não somente a abordagem tradicional de implementação da estratégia, ao incorporar conceitos como Estratégia Emergente proposta por Mintzberg, além de Racionalidade Limitada de Herbert Simon e Aprendizagem de circuito simples e duplo de Argyris e Schön.

Como todo o modelo teórico desenvolvido por Simons (1990, 1991, 1995, 2000) foi construído com base em estudos de casos, isso estimulou diversos pesquisadores a desenvolverem trabalhos empíricos para aferir a validade desse modelo. Isso pode ser visto pelo crescente número de estudos empíricos abordando, integral ou parcialmente, 0 modelo teórico Levers of Control, seja por meio de survey (ABERNETHY e BROWNELL, 1999; HENRI, 2006; WIDENER, 2007, BISBE e OTLEY, 2004; FERREIRA e OTLEY, 2006, NARANJ O-GIL e HARTMANN, 2006, 2007), ou estudos de caso (MARGINSON, 2002; BRUINING et al., 2004; COLLIER, 2005; TUOMELA, 2005; KOBER et al., 2007) e também enquanto construto teórico discutido sob uma abordagem metodológica (BISBE, BATISTA-FOGUET e CHENHALL, 2007).

Uma pesquisa efetuada em janeiro de 2008 nas bases do Congresso USP de Controladoria e Contabilidade, ENANPAD, Revista Contabilidade e Finanças, RAE e RAC não identificou nenhum artigo empírico relacionado ao tema. Apenas um trabalho de Silva, Silva e Corso (2007) utilizou o construto no Brasil, porém, com foco na Estratégia.

Tendo como base a quantidade de estudos na literatura internacional sobre o modelo, este trabalho tem como objetivo identificar quais contribuições os estudos sobre o modelo teórico Levers of Control de Simons podem trazer para a pesquisa em Contabilidade Gerencial no Brasil.

O trabalho tem como principais contribuições analisar o modelo teórico, identificar e consolidar os resultados empíricos acerca do construto e sugerir pesquisas que possam explorá-lo. A metodologia utilizada é uma análise bibliográfica com discussão dos resultados 
das pesquisas.

Estudos que analisam a produção da literatura sobre determinado tema são comumente publicados nos principais periódicos internacionais de Contabilidade, existindo até periódicos específicos como o Journal of Accounting Literature. Assim, este estudo pode gerar contribuições para os pesquisadores brasileiros em Contabilidade Gerencial ao organizar e discutir os resultados das pesquisas sobre um construto que vem crescentemente sendo explorado na literatura internacional.

Esse trabalho se inicia por esta seção, a seção 2 trata do referencial teórico de Simons, a seção 3 trata do desenvolvimento da pesquisa, por fim, na seção 4 são feitas as considerações finais e sugestões para futuras pesquisas.

\section{2 - REFERENCIAL TEÓRICO LEVERS OF CONTROL}

Na visão de Simons (1995), em seu modelo teórico Levers of Control, existem quatro tipos de sistemas de controle: (i) Sistemas de Crenças (Belief Systems), (ii) Sistemas de Restrições (Boundary Systems), (iii) Sistemas de Controle Diagnóstico (Diagnostic Control Systems) e (iv) Sistemas de Controle Interativo (Interactive Control Systems). A construção desses quatro sistemas tem como pano de fundo o conceito de Tensões Dinâmicas geradas pelo uso do SCG, as quais ocorrem quando os gerentes têm que atingir objetivos organizacionais que podem ser conflitantes, mas que a organização deseja certa complementaridade e um balanceamento entre esses objetivos (HENRI, 2006). O propósito do SCG é gerenciar as tensões organizacionais, as quais são similares ao conceito de paradoxos ou dilemas, e são defendidas por alguns autores como importantes para garantir a efetividade organizacional (QUIN, 1988 apud VAN DE VEN e ENGLEMAN, 2004). Assim, a idéia é que os quatro sistemas ajudem no balanceamento das tensões organizacionais, produzindo um adequado equilíbrio (SIMONS, 1995).

Uma das Tensões Dinâmicas diz respeito ao tempo dos executivos. Simons (1995) argumenta que há uma atenção limitada dos executivos em razão de eles não terem o tempo necessário para dar atenção a todo o SCG; como conseqüêencia, os executivos ficam responsáveis por apenas parte do SCG, enquanto que o monitoramento da outra parte do SCG é delegado aos subordinados (SIMONS, 1990). Por conta da falta de tempo, a alta direção cuida pessoalmente das variáveis mais críticas, as quais refletem as incertezas estratégicas, e que geram impacto na posição competitiva da empresa.

O quadro 1 resume os quatro tipos de sistema de controle proposto por Simons e reproduzido em uma obra que consolidou o construto (SIMONS, 2000).

\begin{tabular}{|l|c|c|c|}
\hline \multicolumn{1}{|c|}{ Sistema de Controle } & Propósito & Objeto de informação & $\begin{array}{c}\text { Controle da Estratégia } \\
\text { como }\end{array}$ \\
\hline Sistemas de Crenças & $\begin{array}{c}\text { Empreender e buscar } \\
\text { expandir a atividade }\end{array}$ & Visão & Perspectiva \\
\hline Sistema de Restrições & $\begin{array}{c}\text { Prover limites para 0 } \\
\text { campo de ação }\end{array}$ & $\begin{array}{c}\text { Area estratégica de } \\
\text { atuação }\end{array}$ & Posição Competitiva \\
\hline $\begin{array}{l}\text { Sistemas Diagnósticos } \\
\text { de Controle }\end{array}$ & $\begin{array}{c}\text { implementar as } \\
\text { estratégias planejadas }\end{array}$ & Planos e metas & Plano \\
\hline $\begin{array}{l}\text { Sistemas Interativos de } \\
\text { Controle }\end{array}$ & $\begin{array}{c}\text { Estimular e guiar } \\
\text { estratégias emergentes }\end{array}$ & Incertezas Estratégicas & Padrão de ações \\
\hline
\end{tabular}

Quadro 1 - Escopo dos Sistemas de Controle - Levers of Control

Fonte: SIMONS, 2000, p. 304 


\section{1 - SISTEMA DE CRENÇAS - BELEFS SYSTEM}

Simons (1995, p. 3) define sistema de crenças como um "conjunto explícito de definições organizacionais que a alta direção comunica formalmente e reforça sistematicamente para uniformizar valores, propósitos e direção para a organização".

O exemplo dado por Simons (1995) é a declaração de Missão, Visão e Valores da empresa J ohnson \& J ohnson e, comumente, divulgada pelas grandes empresas. Entretanto, para que, efetivamente, funcionem como norteadores de comportamento organizacional é importante que as atitudes praticadas sejam coerentes com as crenças divulgadas e assim possam orientar as decisões nos casos omissos pelo código de ética.

O Sistema de Crenças deve influenciar tanto os chamados Controles Pessoais, que é quando a própria pessoa controla o seu próprio comportamento, quanto o Controle Cultural, em que um conj unto de crenças compartil hadas funciona como um padrão de comportamento para os demais membros organizacionais (MERCHANT e VAN DER STEDE, 2007).

\section{2 - SISTEMA DE RESTRIÇÕES - BOUNDARY SYSTEMS}

Já o Sistema de Restrições tem como propósito estabelecer limites para busca de oportunidades baseado em conceitos de riscos (SIMONS, 1995, p. 39). O Sistema de Restrições auxilia os gestores na medida em que delimita o nível de risco que a organização está disposta a assumir, sendo operacionalizado pela divulgação de indicadores de endividamento, grau de alavancagem operacional e financeira, receitas e ativos. A definição do mercado de atuação e também dos produtos e serviços oferecidos contribui para evitar a perda de tempo na busca de oportunidades que não são o foco da organização.

Esse sistema também é operacionalizado pelos Códigos de Conduta existentes em grandes organizações e pelo conjunto de normas e procedimentos, muitas delas incluídas na forma de sistemas automatizados de controle e de gestão dos riscos vinculados ao negócio.

\section{3 - SISTEMAS DIAGNÓSTICOS DE CONTROLE - DIAGNOSTIC CONTROL SYSTEMS}

Para Simons (1995, p. 59), "sistemas de Controle Diagnóstico são os sistemas de informação formais que os gerentes usam para monitorar os resultados organizacionais e corrigir desvios em relação ao padrão definido de desempenho".

O sistema de controle diagnóstico ou Uso Diagnóstico é o uso tradicional do sistema de controle gerencial, o qual tem como propósito avaliar a implementação das estratégias planejadas (SIMONS, 2000). O Uso Diagnóstico é focado na implementação das estratégias planejadas e na orientação da alta direção quando os resultados não estão de acordo com os planos (SIMONS, 1991), possibilitando o gerenciamento por exceção calcado na comparação com o orçamento (MERCHANT e VAN DER STEDE, 2007).

Diversas críticas são feitas ao Uso Diagnóstico, especialmente em relação à visão que os números contábeis refletem apenas o desempenho de curto prazo e geram uma orientação para curto prazo (MERCHANT, 1990; VAN DER STEDE, 2000); ou seja, o foco é atingir metas de resultados econômicos, mesmo prejudicando objetivos de longo prazo. Discute-se, nesse ponto, que isso impediria o processo de empreendedorismo e inovação, já que os gestores se sentiriam prejudicados se tivessem que gastar mais do que os valores orçados, 
mesmo que fosse necessário para melhorar o desempenho no médio e longo prazos. Todos esses argumentos reforçam a visão de que num ambiente dinâmico as medidas contábeis, principalmente as derivadas do processo orçamentário, não podem servir como única base para avaliação de desempenho (OSTERLOH e FREY, 2002).

\section{4 - SISTEMAS DE CONTROLE INTERATIVO - INTERACTIVE CONTROL SYSTEMS}

Simons (1991, p. 52) afirma que "reconhecer que o mesmo sistema pode ser classificado como interativo em uma empresa e diagnóstico em outra empresa é importante para o entendimento dos resultados do seu trabalho."

O autor ilustra com o exemplo da elaboração do orçamento na J ohnson $\&$ J ohnson que era utilizado de forma interativa, enquanto que em outras empresas ( 19 empresas foram pesquisadas) o orçamento era usado somente diagnosticamente, já que não era revisado durante o decorrer do ano e era monitorado por grupos de assistentes e reportados aos diretores somente por exceção. Simons (2000, p. 142) exemplifica que o orçamento, o relatório de participação de mercado ou mesmo o balanced scorecard podem ser usados tanto diagnosticamente como interativamente. Simons (1995) afirma que os Sistemas Interativos de Controle têm as seguintes características:

- A informação gerada por esse sistema é um importante e recorrente tema pela alta direção;

- Demandam freqüente e regular atenção dos gerentes operacionais de todos os níveis da organização;

- Os dados gerados por esse sistema são interpretados e discutidos em reuniões presenciais com os superiores, subordinados e pares;

- O sistema é um catalisador para desafio contínuo e debate sobre os dados, premissas e planos de ação

- Fornecer informações sobre os efeitos das incertezas estratégicas sobre a estratégia da empresa. (SIMONS, 1995, p. 95; p. 108)

Quanto ao aspecto de quem o utiliza, Simons (2000) define que os Sistemas Interativos de Controle são os sistemas de informações formais que os gerentes usam para pessoalmente envolver-se nas decisões das atividades de seus subordinados.

\section{5 - INCERTEZAS ESTRATÉGICAS}

Sobre as incertezas estratégicas, objeto do Uso Interativo, Simons (2000) qualifica que estas são diferentes das variáveis críticas de desempenho. Estas últimas, enumeradas em balanced scorecards ou outros sistemas diagnósticos de controle, são calcadas nos planos e metas. Para ele incertezas estratégicas são "as ameaças e oportunidades emergentes que podem invalidar as premissas sobre as quais a estratégia corrente é baseada" (SIMONS, 2000, p. 215).

Pode-se operacionalizar como Incertezas Estratégicas os indicadores sobre Concorrentes, Tecnologia, Produtos Substitutos, Fatores Ambientais que influenciam os resultados e a situação financeira, tais como Taxa de Juros, Taxa de Câmbio, Inflação, comportamento do PIB, etc.. Já a classificação da importância das Incertezas Estratégicas 
para as empresas deve levar em conta a dificuldade de prever o comportamento dessas variáveis, e também os diferentes graus de influência sobre o desempenho da empresa. Assim, incertezas com maior dificuldade de previsão e maior influência sobre o desempenho seriam mais importantes e, portanto, passíveis de um monitoramento mais cuidadoso, sob uma abordagem contingencial.

\section{6 - ANÁLISE DOS SISTEMAS}

O modelo Levers of Control, como todo modelo, é uma simplificação da realidade, assim, torna-se necessário uma reflexão crítica sobre os componentes desse modelo.

A respeito da aderência à realidade empresarial nota-se que os Sistemas de Restrições e de Crenças estão presentes e materializados principalmente nas organizações de grande porte, e espera-se que as pesquisas mostrem esses dois sistemas funcionando de forma balanceada. Outro resultado esperado é que se evidencie a utilização do Sistema de Crenças como forma de divulgação da cultura e dos valores, influenciando o comportamento das pessoas e estimulando o controle pessoal e o controle cultural. Interativo.

No quadro 2 busca-se resumir as principais diferenças entre o Uso Diagnóstico e Uso

\begin{tabular}{|c|c|c|}
\hline Dimensão & Uso Diagnóstico & Uso Interativo \\
\hline $\begin{array}{c}\text { Quanto à atenção da alta } \\
\text { direção }\end{array}$ & $\begin{array}{c}\text { Pouca, delega para os } \\
\text { subordinados }\end{array}$ & Muita, envolve-se pessoalmente \\
\hline Quanto às estratégias & Estratégias planejadas & Estratégias Emergentes \\
\hline Quanto ao resultado & $\begin{array}{c}\text { Correção de desvios } \\
\text { Aprendizado de Circuito } \\
\text { Simples }\end{array}$ & $\begin{array}{c}\text { Formulação de novos planos de } \\
\text { ação } \\
\text { Aprendizado de Circuito Duplo }\end{array}$ \\
\hline $\begin{array}{c}\text { Quanto ao escopo } \\
\text { Quanto à freqüência } \\
\text { de interações com os } \\
\text { subordinados }\end{array}$ & $\begin{array}{c}\text { Diálogo esporádico } \\
\text { Gestão por exceção }\end{array}$ & $\begin{array}{c}\text { Diálogo contínuo } \\
\text { Agenda recorrente }\end{array}$ \\
\hline $\begin{array}{c}\text { Quanto ao uso nas } \\
\text { camadas organizacionais }\end{array}$ & Delegado ao "staff" & Múltiplos níveis gerenciais \\
\hline
\end{tabular}

\section{Quadro 2 - Principais Diferenças entre o Uso Diagnóstico e Uso Interativo}

Fonte: elaborado pelos autores com base em Simons (1995, 2000)

Com base no quadro 2, identifica-se que diversas teorias compõem as dimensões que diferenciam Uso Diagnóstico de Uso Interativo. A Racionalidade Limitada proposta por Simon está presente na questão da atenção da alta direção, reconhecendo que este nível organizacional toma como foco as incertezas estratégicas, embora isso possa ser explicada por variáveis organizacionais como estrutura hierárquica, nível de autonomia e disponibilidade das informações.

A questão da emergência das estratégias (MINTZBERG e LAMPEL, 1999) posiciona o Uso Interativo como influenciador desse fenômeno, habilitando-o a se configurar como um recurso sob a perspectiva da Resources-Based View (HENRI, 2006). Aspectos ligados ao processo decisório também são abordados, como a teoria do Circuito Simples e Circuito Duplo proposto por Argyris e Schön (1996). 
Quanto ao aspecto da comunicação entre os gestores também é destacado por meio do diálogo esporádico versus contínuo, embora o modelo não discorra sobre a questão dos controles formais versus informais do controle gerencial, consoante o proposto por Merchant e Van Der Stede (2007).

Quanto à abordagem contingencial, é esperado que os resultados das pesquisas indiquem que organizações sujeitas a maiores incertezas estratégicas tenham maior intensidade do Uso Interativo, enquanto que o Uso Diagnóstico deveria ser menos intenso neste tipo de organizações.

Outro resultado esperado é a discussão de que é muito difícil estabelecer uma fronteira temporal dos usos, ou seja, é esperado identificar que estes estão em um fluxo contínuo na rotina organizacional.

\section{3 - DESENVOLVIMENTO DA PESQUISA. ANÁLISE DOS ESTUDOS QUE UTILIZARAM 0 REFERENCIAL TEÓRICO DE SIMONS}

Foram analisados os trabalhos que utilizaram, mesmo que parcialmente, o modelo Levers of Control de Simons. Para isso fez-se uma busca, cuj o término se deu em janeiro de 2008, em três dos principais periódicos de Contabilidade Gerencial (Accounting, Organizations and Society, J ournal of Management Accounting Research, Management Accounting Research) os quais estão presentes nesse tipo de pesquisa bibliográfica, no principal periódico de Estratégia (Strategic Management Journal). Fez-se também uma pesquisa no SSRN com as seguintes palavras-chave: levers of control, simons, interactive control, management control and strategy. 0 método de análise utilizado foi ler primeiramente o resumo para confirmar se pelo menos partes do modelo foram abordadas, e em caso positivo, os trabalhos foram lidos integralmente. Porém, muitos trabalhos tinham outros obj etivos concomitantes e, nesses casos, a conclusão relatada no presente trabalho limitou-se ao modelo Levers of Control. A seguir são relacionados 16 trabalhos, em ordem cronológica, destacando-se o tipo de estudo, a validação do modelo teórico e os principais resultados.

1. TANI, T. Interactive control in target cost management. Management Accounting Research, 6, p. $399-414,1995$.

Tani estudou a gestão de custo meta sob a ótica da visão da engenharia simultânea. O trabalho analisou 109 empresas japonesas listadas na Bolsa de Tóquio e que utilizam o custo meta, uma ferramenta constante do que se convencionou chamar-se de Strategic Management Accounting (CADEZ e GUILDING, 2008).

No tocante ao construto Uso Interativo, os resultados mostraram que este ocorre nos encontros de gerentes departamentais, principalmente pela discussão do planejamento de desenvolvimento de produtos e nas reuniões de análise de custos, sendo esses eventos essenciais para o compartilhamento de informações. Outro aspecto que validou a teoria do Uso Interativo foi a evidência de que para se adaptar às inovações tecnológicas e às diferentes necessidades dos clientes, as organizações monitoravam esses aspectos interativamente.

O trabalho também identificou que a alta direção interage mais com os gerentes funcionais nas reuniões de planejamento de produto do que nas reuniões de custos, provavelmente para identificar informações para formulação da estratégia, confirmando que - Uso Interativo se relaciona mais com as incertezas estratégicas e que o Uso Diagnóstico é delegado aos gestores subordinados. 
2. VANDENBOSHC, B. An empirical analysis of the association between the use of executive support systems and perceived organizational competitiveness. Accounting, Organizations and Society, 24, p. 77 - 92, 1999.

O trabalho de Vandenbosch (1999) foi o primeiro a testar empiricamente aspectos mais abrangentes do modelo teórico de Simons. As questões de pesquisa do trabalho se voltaram para identificar para quais usos a informação estava direcionada e como essa utilização estava relacionada com melhoria da competitividade. Os respondentes totalizaram 344 em 25 organizações. Os construtos analisados foram denominados em score keeping, improving understanding, focusing attention, legitimizing decision e enabling competitiveness. No construto score keeping as perguntas giraram em torno de como o uso do SCG permitia monitorar resultados e rever as medidas-chave. Já em focusing attention as perguntas procuraram verificar o uso do SCG para monitorar fatores críticos de sucesso e prover uma visão comum da organização.

Destaca-se o fato de ter sido um dos trabalhos pioneiros e que o questionário desenvolvido foi utilizado nos estudos seguintes. Também se concluiu que o Uso Interativo está mais relacionado com a percepção de melhoria de desempenho. Como restrições há o fato do construto desempenho ter sido observado apenas como uma percepção, embora justificável pelo fato de serem gestores mais distantes dos números contábeis, e também a pequena quantidade de organizações.

3. ABERNETHY, M.A.; BROWNELL, P. The role of budgets in organizations facing strategic change: an exploratory study. Accounting, Organizations and Society, 24, p. 189-204, 1999.

O trabalho de Abernethy e Brownell (1999) utilizou a técnica estatística Regressão Linear Múltipla para identificar o relacionamento entre desempenho e mudança de orientação estratégica, moderado pela extensão com que os orçamentos são usados interativamente. As principais restrições relatadas pelos autores foram que a amostra foi constituída somente de 63 hospitais públicos na Austrália e o fato de o respondente ocupar o cargo de maior nível hierárquico.

A conclusão do estudo é coerente com a fundamentação de Simons que a alta direção seleciona diferentes controles para utilizar interativamente, dependendo da natureza das incertezas estratégicas (ABERNETHY e BROWNELL, 1999, p.200). Como ponto forte destacase a contribuição de corroborar a teoria de Simons, ao mostrar que um determinado artefato pode ser utilizado tanto de forma diagnóstica como de forma interativa, além da questão do Uso Interativo pela alta direção.

4. MARGINSON, D. E. W. Management control systems and their effects on strategy formation at middle-management levels: evidence from a UK organization. Strategic Management J ournal. 23, 11, p. 10 - 19, Nov. 2002.

Marginson (2002) fez um estudo de caso longitudinal em uma empresa britânica de telecomunicações caracterizada por inovações e pela criatividade dos gerentes de nível médio. Essa empresa estava passando por um programa de mudanças estratégicas com implementação de Balanced Scorecard. O estudo confirmou que: (i) a alta direção usa o sistema de crenças e valores para incentivar a mudança estratégica; (ii) os controles podem ser usados pelos gestores de todos níveis hierárquicos para assegurar a implementação da estratégia; (iii) uma parcela dos indicadores críticos de desempenho pode ser usada para assegurar um padrão mínimo de desempenho em áreas importantes do processo estratégico. (MARGINSON, 2002). Outro resultado importante é o fato de validar o papel do Sistema de Crenças como indutor do comportamento organizacional. 
Esse trabalho tem ainda o mérito de ter sido publicado no mais importante periódico sobre Estratégia, o que contribui para a difusão do Modelo para leitores e pesquisadores de outra área bastante próxima.

5. BISBE, J .; OTLEY, D. The effects of the interactive use of management control systems on product innovation. Accounting, Organizations and Society, 29, p. 709 - 737, 2004.

Bisbe e Otley (2004) estudaram os efeitos do Uso Interativo do sistema de controle gerencial na inovação de produtos, com o propósito de explicitar os diferentes efeitos do Uso Interativo na inovação de produto (variável moderadora) e no desempenho (medido por auto-avaliação) em 40 indústrias espanholas de médio porte (os respondentes foram os presidentes). Foram testados os argumentos de Simons no tocante às afirmações que o Uso Interativo estimula a experimentação, a busca de oportunidades e o surgimento de novas iniciativas (BISBE e OTLEY, 2004).

A hipótese de que maior Uso Interativo do SCG pela alta direção implica maior inovação de produtos não foi validada, somente em empresas com baixa taxa de inovação essa hipótese foi validada. Também não foi validada a hipótese de que existe um relacionamento indireto positivo entre o Uso Interativo e o desempenho ativado por meio da inovação de produto, entretanto foi validada a hipótese que o maior uso pela alta direção implica em maior efeito da inovação de produto no desempenho.

Destaca-se a contribuição de que o construto Levers of Control tem maior ou menor aderência dadas as características contingenciais de cada empresa, confirmando que esse modelo é apropriado para estudos que utilizem a abordagem contingencial.

6. BRUINING, H.; BONNET, M.; MIKE WRIGHT, M. Management control systems and strategy change in buyouts. Management Accounting Research, 15, p. 155 - 177, 2004.

Bruining et al (2004) examinaram por meio de dois estudos de casos em empresas adquiridas pela gerência (Management Buy-Outs MBO), porém, situadas em dois diferentes contextos de estratégia: uma empresa recém privatizada com foco em inovação, e outra empresa com escopo mais restrito para inovação. O trabalho examinou o sistema de controle gerencial antes e durante a alienação do controle societário.

Os autores concluem que a MBO é uma oportunidade para mudança no sistema de crenças, no Uso Interativo do SCG e nas mudanças estratégicas. Eles ressaltam a importância das capacidades na ótica da Visão Baseada em Recursos (VBR) ao afirmarem que a interação entre investidor e agentes externos pôde gerar importantes contribuições para o aprendizado por meio da geração de idéias valiosas para elaborar estratégias em ambientes de mudanças no mercado. Como contribuição relevante tem-se a validação dos benefícios do uso do controle interativo e a ressalva de que é necessária a presença de profissionais competentes para usufruir adequadamente o SCG.

7. TUOMELA, T-S. The interplay of different levers of control: A case study of introducing a new performance measurement system. Management Accounting Research, 16, p. $293-320$, 2005.

Tuomela (2005) fez um estudo de caso longitudinal (período de quatro anos) sobre a implementação de um novo sistema de mensuração de desempenho em uma subsidiária de uma grande empresa finlandesa. 0 estudo concluiu que o sistema de controle gerencial pode ser utilizado de forma diagnóstica e na forma interativa e que a forma interativa 
propicia a melhoria da qualidade da gestão e aumenta o comprometimento dos gestores com as metas estratégicas. O construto Levers of Control tem implicações em todos os sistemas de controle e o Uso Interativo tem maiores desafios na implementação, porém, com maiores benefícios, quando comparado com o Uso Diagnóstico. Por outro lado, o Uso Interativo consumiu mais tempo dos gerentes em quantidade de reuniões e a duração das mesmas também aumentou (TUOMELA, 2005), assim, há que se balancear a tensão entre escassez de tempo e interatividade.

8. COLLIER, P.M. Entrepreneurial control and the construction of a relevant accounting. Management Accounting Research, 16, p. 321 - 339, 2005.

Collier (2005) fez um estudo de caso único longitudinal em uma pequena empresa australiana, porém com atuação internacional. O foco do estudo de Collier (2005) foi estudar a contribuição do framework de Ferreira e Otley (2006), o qual incorpora partes do framework de Simons (1995), porém, com destaque para a influência do empreendedor na construção desse sistema de controle gerencial.

O estudo mostrou que o sistema de controle gerencial não era construído com base em informações contábeis (sequer a empresa possuía um contador em seus quadros profissionais), mas apenas calcado em uma planilha que continha informações monetárias como fluxo de caixa, capacidade de endividamento e informações não monetárias como participação de mercado. O controle gerencial era exercido pelo fundador da empresa de forma interativa, por meio de reuniões informais com os gerentes em eventos extra-horário de trabalho.

Este estudo contribuiu para diminuir a visão de que o framework Levers of Control é somente aplicável em grandes organizações, e demonstra as variáveis que são importantes para o controle gerencial de uma organização, mesmo sem a presença formal de um contador. Collier (2005) também mostra a importância da comunicação entre proprietário e gestores para o monitoramento de desempenho, porém, considera que o modelo de Simons dedica pouca atenção aos controles sociais e culturais.

9. FERREIRA, A.; OTLEY, D. Exploring inter and intra-relationships between the design and use of management control systems. SSRN. 2006.

Ferreira e Otley (2006) estudaram os relacionamentos entre as dimensões desenho e uso do SCG nas 1.000 maiores empresas portuguesas não-financeiras (122 respondentes). A dimensão desenho foi baseada principalmente no trabalho de Chenhall e Morris de 1986 e a dimensão uso foi baseada no trabalho de Simons (1995). O estudo teve como variável dependente o Uso Disfuncional, e como variáveis independentes, o Desenho, Uso Interativo e o Uso Diagnóstico. O método estatístico foi a Análise de Regressão Múltipla, com análise fatorial confirmatória.

O estudo indicou que Uso Diagnóstico do SCG tem maior impacto no Uso Disfuncional do que o Uso Interativo, e a justificativa é que o Uso Interativo tende a ser mais positivo e inspirador do que o Uso Diagnóstico, que tende a ser mais negativo e repressor. Os autores também afirmam que a discussão face a face com os gerentes pode reduzir a constituição de reservas orçamentárias. Outro achado importante é que a forma de remuneração variável não explica o comportamento disfuncional, contrariando a literatura (FERREIRA e OTLEY, 2006).

10. FROW, N.; MARGINSON, D.; OGDEN, S. Encouraging strategic behaviour while maintaining management control: Multi-functional project teams, budgets, and the negotiation of 
shared accountabilities in contemporary enterprises. Management Accounting Research, 16, p. 269 - 292, 2006.

Frow, Marginson e Ogden (2006) estudaram uma empresa multinacional do setor de tecnologia com foco na accountabil ity e discutiram asformas de controle social na organização, principalmente quanto à questão da sua formalização e também de como esses controles sociais são importantes para trabalhar com a accountability nos níveis hierárquicos.

Restringindo-se ao foco da teoria de Simons, os achados mostram que os gerentes preferem resolver os problemas por meio de contato pessoal por entenderem ser mais eficiente, e também que as formas de controle social são reforçadas pelos procedimentos formais de controle, facilitando a cooperação informal entre os gerentes. Essa conclusão é coerente com a idéia de que o Uso Interativo e Uso Diagnóstico são complementares (HENRI, 2006).

11. NARANJ O-GIL, D. , HARTMANN, F. How Top Management Teams Use Management Accounting Systems to Implement Strategy. J ournal of Management Accounting Research, 18, p. 21 - 53, 2006.

Naranjo-Gil e Hartmann (2006) testaram os relacionamentos entre nível de profissionalismo da alta direção e o Uso Diagnóstico e Interativo do SCG, relacionando com a utilização de medidas de desempenho financeiras $\mathrm{x}$ não financeiras, e também o relacionamento entre essas variáveis e dois tipos de estratégia (liderança em custo e flexibilidade). A hipótese estudada foi que quanto maior é o nível de profissionalismo, maior o Uso Interativo, e quanto maior o uso de medidas não financeiras, maior a ênfase na estratégia de flexibilidade.

Os resultados do estudo, usando a Modelagem de Equação Estrutural por meio da estimação Partial Least Squares (PLS), mostraram que: (i) Uso Diagnóstico não está relacionado positivamente com estratégia de baixo custo, o que enseja que a ativação desse tipo de orientação estratégica possa ser feita por diferentes usos do SCG; (ii) os resultados validam a afirmação de Simons que um estilo interativo promove inovação; (iii) também o estudo mostrou que diferentes usos do SCG provocam diferentes efeitos na estratégia.

12. NARANJ O-GIL, D., HARTMANN, F. Management accounting systems, top management team heterogeneity and strategic change. Accounting, Organizations and Society. v. 32, 7 8, Oct.-Nov. 2007.

Naranjo-Gil e Hartmann (2007) estudaram os relacionamentos entre o Uso Interativo e o Uso Diagnóstico do SCG como moderadores da relação entre a heterogeneidade da alta direção e o comportamento estratégico da organização. 0 estudo foi feito com a alta direção de 103 hospitais públicos da Espanha e contou com respondentes válidos no total de 383.

Os principais achados do estudo, restringindo-se ao construto de Simons foram: (i) a heterogeneidade da alta direção é positivamente relacionada com o Uso Interativo do SCG; (ii) o Uso Interativo do SCG não está positivamente relacionado com hospitais que estão se movendo para uma posição defender, mas é positivamente relacionado com aqueles que estão buscando uma posição prospector; (iii) existe um relacionamento entre o escopo do SCG e o Uso Interativo do SCG. Prospector e defender são tipologias estratégicas propostas por Miles e Snow em 1978. Defender está em ambiente estável e sob uma estrutura centralizada, enquanto que prospector está em ambiente instável e busca a inovação dentro de uma estrutura organizacional flexível (SIMONS, 1990). 
13. HENRI, J. F. Management Control Systems and Strategy: a Resource-based Perspective. Accounting, Organizations and Society, 31, p. 529 - 558, 2006.

O trabalho de Henri (2006) estudou partes do construto de Simons, com foco no Uso Interativo, Uso Diagnóstico e Tensão Dinâmica. A inovação do trabalho foi relacionar esses construtos com a Estratégia, especificamente a VBR, e como variável dependente, o desempenho medido por auto-avaliação. Henri realizou um survey com 383 respondentes da alta direção de empresas canadenses (unidades de negócio ou empresas independentes) e utilizou Modelagem de Equação Estrutural com o método de estimação LISREL.

Henri (2006) defende a complementaridade do Uso Diagnóstico com o Uso Interativo, pois o resultado do seu trabalho mostrou que o Uso Diagnóstico exerce pressão negativa sobre as capacidades (orientação para mercado, empreendedorismo, inovação e aprendizado organizacional), enquanto o Uso Interativo favorece as capacidades de orientação para mercado, empreendedorismo, inovação e aprendizagem organizacional. A inovação do estudo está na introdução do construto Tensões Dinâmicas, mostrando que este construto, influenciado pelo Uso Diagnóstico e Uso Interativo, produz efeitos positivos no desenvolvimento das competências, habilitando o SCG como um recurso valioso, distintivo e imperfeitamente imitável dentro da ótica Visão Baseada em Recursos (BARNEY, 2007).

Esses resultados sugerem que o uso tradicional do controle gerencial contribui negativamente para o desenvolvimento das competências, as quais são a base para obtenção de desempenho, o que está alinhado com os estudos de Merchant (1990) e Van Der Stede (2000), ou seja, o Uso Diagnóstico contribui para um desenvolvimento de uma orientação para curto prazo, com o gestor comportando-se disfuncionalmente. Porém, estes resultados podem ser diferentes em países com cultura distinta das dos países estudados.

14. WIDENER, S. K. An empirical analysis of the levers of control framework. Accounting, Organizations and Society, 32, p. 757 - 788, 2007.

Widener (2007) testou os relacionamentos entre dois elementos estratégicos (incerteza estratégica e risco estratégico), os quatro componentes do Sistema de Controle (Sistema de Crenças, Sistema de Restrições, Sistemas Diagnósticos de Controle e Sistemas Interativos de Controle), custo e benefícios do uso (atenção da alta direção foi classificada como custo de utilização, aprendizado foi classificado como benefício), e desempenho. O survey realizado por Widener abrangeu 122 Diretores financeiros de empresas norte-americanas com receitas anuais médias de 282 milhões de dólares, e na análise dos dados foi utilizada a modelagem de equação estrutural com método de estimação LISREL.

Widener (2007) identificou que orientação para aprendizagem organizacional e atenção gerencial estão associadas ao desempenho (medido em dois modelos, por autoavaliação e também por ROA), o que é coerente com a teoria estudada de que aprendizagem influencia o desempenho. Outra contribuição de Widener (2007, p.783) é que o Uso Interativo do sistema de controle gerencial não está positivamente associado com Aprendizagem Organizacional, sendo que este ativa a Aprendizagem Organizacional por meio do Sistema Diagnóstico de Controle. Muitos dos controles abrangidos pelo modelo Levers of Control são interdependentes e complementares, sendo que o Sistema Interativo é interdependente com os Sistemas Diagnósticos e Sistemas de Crenças (WIDENER, 2007, p. 782).

O estudo de Widener (2007) contribui para a literatura já que os resultados se contrapõem, parcialmente, aos resultados de Henri (2007) no tocante ao efeito negativo do Uso Diagnóstico. O possível viés do estudo é que os respondentes são diferentes do estudo de Henri (2007), porém, o resultado acerca da aprendizagem é coerente com literatura que afirma que o Uso Diagnóstico influencia as decisões de circuito simples (SIMONS, 1995, 2000), uma das formas de operacionalização do construto Aprendizagem Organizacional. 
15. KOBER, R.; NG, J.; PAUL, B.J. The interrelationship between management control mechanisms and strategy. Management Accounting Research, 18, p. 425 - 452, 2007.

Kober et al. (2007) testaram a relação entre o SCG e a estratégia em um centro de pesquisas médicas australiano, em um estudo de caso único longitudinal que envolveu revisão de documentos, entrevistas e questionários.

A principal conclusão do estudo foi que o Uso Interativo do SCG contribui para facilitar uma mudança na estratégia e que, quando a mudança na estratégia ocorre, o mecanismo do SCG também muda para se adequar a esta mudança (KOBER et al. 2007), sendo validada a teoria contingencial onde a estrutura é decorrente da estratégia.

Observou-se ainda um aumento do uso do SCG no monitoramento de resultados e controle de custos de maneira interativa e que isso facilitou a mudança na estratégia, ou seja, evidenciou-se o papel ativo do Uso do SCG na formulação da estratégia, e não somente na implementação. Portanto, foi suportada a teoria que novas estratégias podem surgir por meio do Uso Interativo.

16. BISBE, J.; BATISTA-FOGUET, J. M; CHENHALL, R. Defining management accounting constructs: A methodological note on the risks of conceptual misspecification. Accounting, Organizations and Society, 32, p. 789 - 820, 2007.

Bisbe, Batista-Foguet e Chenhall (2007) discutiram a questão epistemológica dos construtos em Contabilidade Gerencial. O objetivo do trabalho foi alertar para os riscos de mensurar construtos de forma inadequada, o que foi ilustrado por meio da adoção do construto Uso Interativo de Simons.

Na visão de Bisbe et al. (2007, p.795-796) o Uso Interativo abrange outros construtos como intensidade da comunicação, aprendizado de circuito duplo de Argyris e Schon de 1978, rigidez ou flexibilidade no estilo de avaliação de desempenho de Merchant de 1985 e Van der Stede de 2001, ou seja, todas essas dimensões são necessárias para que o construto Uso Interativo seja conceitualmente válido. Após uma extensa análise dos trabalhos de Simons, os autores concluíram que o construto Uso Interativo possui cinco dimensões ou subconstrutos:

(1) uso intensivo pela alta direção;

(2) uso intensivo pelos gestores operacionais;

(3) predominância de desafios e debates face a face;

(4) foco nas incertezas estratégicas;

(5) uma forma não invasiva que facilita e inspira o envolvimento.

Assim, o Uso Interativo é definido como um construto multidimensional emergente, pois é composto por várias dimensões ou sub-construtos, devendo ser modelado em pesquisas futuras como um construto multidimensional emergente formado por dimensões constitutivas (mensuração formativa), ao contrário das pesquisas anteriores que o caracterizaram como um modelo reflexivo. Isso implica que os testes estatísticos devam ser diferentes dos adotados nos estudos como Alpha de Cronbach, Análise Fatorial Confirmatória, dentre outros procedimentos (BISBE et al., 2007).

\subsection{SÍNTESE DOS TRABALHOS ANALISADOS}


No quadro 3, pode-se identificar uma síntese dos trabalhos analisados, evidenciando os principais resultados.

\begin{tabular}{|c|c|c|c|}
\hline Artigo & Tipo de Estudo & $\begin{array}{l}\text { Elemento } \\
\text { do Levers of } \\
\text { Control }\end{array}$ & Principais Resultados \\
\hline Tani (1995) & Levantamento & Uso Interativo & $\begin{array}{l}\text { Uso Interativo ocorre por meio de encontros de gerentes } \\
\text { departamentais, reuniões de análise de custos e } \\
\text { monitoramento. }\end{array}$ \\
\hline $\begin{array}{l}\text { Vandenbosch } \\
\text { (1999) }\end{array}$ & Levantamento & Uso Interativo & $\begin{array}{c}\text { Uso Interativo está mais relacionado com a percepção de } \\
\text { melhoria de desempenho. }\end{array}$ \\
\hline \multirow{2}{*}{$\begin{array}{l}\text { Abernethy } \\
\text { e Brownell } \\
\text { (1999) }\end{array}$} & \multirow{2}{*}{ Levantamento } & \multirow{2}{*}{ Uso Interativo } & $\begin{array}{l}\text { Uso Interativo ocorre por meio da seleção que a alta } \\
\text { direção faz de diferentes controles. }\end{array}$ \\
\hline & & & $\begin{array}{l}\text { Um determinado artefato pode ser utilizado tanto de } \\
\text { forma diagnóstica como de forma interativa. }\end{array}$ \\
\hline \multirow{3}{*}{$\begin{array}{l}\text { Marginson } \\
(2002)\end{array}$} & \multirow{3}{*}{$\begin{array}{l}\text { Estudo de caso } \\
\text { longitudinal }\end{array}$} & \multirow{3}{*}{$\begin{array}{l}\text { Sistema de } \\
\text { Crenças; }\end{array}$} & $\begin{array}{l}\text { Uso do sistema de crenças e valores por parte da alta } \\
\text { direção para incentivar a mudança estratégica. }\end{array}$ \\
\hline & & & $\begin{array}{c}\text { Uso dos controles pelos gestores de todos os níveis } \\
\text { hierárquicos para implementação da estratégia } \\
\text { orqanizacional. }\end{array}$ \\
\hline & & & $\begin{array}{l}\text { Sistema de crenças cumpre um papel de indutor de } \\
\text { comportamento. }\end{array}$ \\
\hline \multirow{3}{*}{$\begin{array}{l}\text { Bisbe e Otley } \\
\qquad(2004)\end{array}$} & \multirow{3}{*}{ Levantamento } & \multirow{3}{*}{ Uso Interativo } & $\begin{array}{l}\text { Maior uso interativo do SCG pela alta direção implica } \\
\text { maior inovação de produtos apenas para empresas com } \\
\text { baixa taxa de inovação. }\end{array}$ \\
\hline & & & $\begin{array}{l}\text { Não foi identificado um relacionamento positivo entre o } \\
\text { Uso Interativo e o desempenho. }\end{array}$ \\
\hline & & & $\begin{array}{c}\text { Maior Uso Interativo pela alta direção implica maior efeito } \\
\text { da inovação de produto no desempenho. }\end{array}$ \\
\hline $\begin{array}{l}\text { Bruining et } \\
\text { al (2004) }\end{array}$ & Estudos de caso & $\begin{array}{l}\text { Sistema de } \\
\text { Crenças e Uso } \\
\text { Interativo }\end{array}$ & $\begin{array}{l}\text { Mudança de controle acionário propicia mudanças no } \\
\text { Sistema de Crenças e na forma do Uso Interativo. Este } \\
\text { requer profissionais com competências adequadas. }\end{array}$ \\
\hline $\begin{array}{l}\text { Tuomela } \\
(2005)\end{array}$ & $\begin{array}{l}\text { Estudo de caso } \\
\text { longitudinal }\end{array}$ & $\begin{array}{l}\text { Uso Diagnóstico } \\
\text { e Uso Interativo }\end{array}$ & $\begin{array}{l}\text { Complementar e o Uso Interativo melhora a gestão e } \\
\text { aumenta o comprometimento dos gestores com as metas } \\
\text { estratégicas, mas é mais dispendioso em termos da } \\
\text { utilizacão do tempo dos gestores. }\end{array}$ \\
\hline Collier (2005) & $\begin{array}{l}\text { Estudo de caso } \\
\text { longitudinal }\end{array}$ & Uso Interativo & $\begin{array}{c}\text { Uso Interativo ocorre por meio de reunióes informais } \\
\text { com gerentes, mesmo sem a presença de contadores na } \\
\text { estrutura organizacional. }\end{array}$ \\
\hline $\begin{array}{l}\text { Ferreira e } \\
\text { Otley (2006) }\end{array}$ & Levantamento & $\begin{array}{l}\text { Uso Diagnóstico } \\
\text { e Uso Interativo }\end{array}$ & $\begin{array}{l}\text { Uso Diagnóstico do SCG tem maior impacto no uso } \\
\text { disfuncional do que o uso interativo. }\end{array}$ \\
\hline $\begin{array}{l}\text { Frow et al } \\
\quad(2006)\end{array}$ & Estudo de caso & $\begin{array}{l}\text { Uso Diagnóstico } \\
\text { vs. Uso } \\
\text { Interativo } \\
\end{array}$ & Uso Interativo e Uso Diagnóstico são complementares. \\
\hline \multirow{3}{*}{$\begin{array}{l}\text { Naranjo-Gil } \\
\text { e Hartmann } \\
\quad(2006)\end{array}$} & \multirow{3}{*}{ Levantamento } & \multirow{3}{*}{$\begin{array}{l}\text { Uso Diagnóstico } \\
\text { vs. Uso } \\
\text { Interativo }\end{array}$} & $\begin{array}{c}\text { Uso Diagnóstico não está relacionado positivamente com } \\
\text { estratégia de baixo custo. }\end{array}$ \\
\hline & & & Uso Interativo promove inovação. \\
\hline & & & $\begin{array}{c}\text { Diferentes usos do SCG provocam diferentes efeitos na } \\
\text { estratégias. }\end{array}$ \\
\hline \multirow{2}{*}{$\begin{array}{l}\text { Naranjo-Gil } \\
\text { e Hartmann } \\
\quad(2007)\end{array}$} & \multirow{2}{*}{ Levantamento } & \multirow{2}{*}{$\begin{array}{l}\text { Uso Diagnóstico } \\
\text { vs. Uso } \\
\text { Interativo }\end{array}$} & $\begin{array}{l}\text { Heterogeneidade da alta direção é positivamente } \\
\text { relacionada com o Uso Interativo do SCG. }\end{array}$ \\
\hline & & & $\begin{array}{l}\text { Uso Interativo do SCG é positivamente relacionado com o } \\
\text { tipo estratégico prospector. }\end{array}$ \\
\hline \multirow[t]{2}{*}{ Henri (2006) } & \multirow[t]{2}{*}{ Levantamento } & \multirow{2}{*}{$\begin{array}{l}\text { Uso Interativo, } \\
\text { Uso Diagnóstico } \\
\text { e Tensão } \\
\text { Dinâmica }\end{array}$} & $\begin{array}{c}\text { Uso Diagnóstico e Uso Interativo exercem pressöes } \\
\text { contrárias sobre as capacidades de orientação para } \\
\text { mercado, empreendedorismo, inovação e aprendizagem } \\
\text { organizacional. }\end{array}$ \\
\hline & & & $\begin{array}{c}\text { Os dois usos do SCG são balanceados pela tensão dinâmica } \\
\text { que produz efeitos positivos no desenvolvimento das } \\
\text { competências. }\end{array}$ \\
\hline
\end{tabular}




\begin{tabular}{|c|c|c|c|}
\hline \multirow[b]{2}{*}{$\begin{array}{l}\text { Widener } \\
\text { (2007) }\end{array}$} & \multirow[b]{2}{*}{ Levantamento } & \multirow{2}{*}{$\begin{array}{c}\text { Sistema de } \\
\text { Crenças, } \\
\text { Sistema de } \\
\text { Restrições, } \\
\text { Sistemas } \\
\text { Diagnósticos } \\
\text { de Controle } \\
\text { e Sistemas } \\
\text { Interativos de } \\
\text { Controle }\end{array}$} & $\begin{array}{l}\text { Uso Interativo do sistema de controle gerencial ativa } \\
\text { a aprendizagem organizacional por meio do sistema } \\
\text { diagnóstico de controle. }\end{array}$ \\
\hline & & & $\begin{array}{l}\text { Sistema interativo é interdependente com os sistemas } \\
\text { diagnósticos e sistemas de crenças. }\end{array}$ \\
\hline \multirow{2}{*}{$\begin{array}{l}\text { Kober et al } \\
\quad(2007)\end{array}$} & \multirow{2}{*}{$\begin{array}{l}\text { Estudo de caso } \\
\text { longitudinal }\end{array}$} & \multirow[b]{2}{*}{ Uso Interativo } & $\begin{array}{l}\text { Uso interativo do SCG contribui para facilitar mudança na } \\
\text { estratégia. }\end{array}$ \\
\hline & & & $\begin{array}{c}\text { Uso interativo do SCG na formulação da estratégia, e não } \\
\text { somente na implementação. }\end{array}$ \\
\hline $\begin{array}{l}\text { Bisbe et al } \\
\text { (2007) }\end{array}$ & Metodológico & Uso Interativo & $\begin{array}{l}\text { Uso Interativo possui cinco dimensões: (1) uso intensivo } \\
\text { pela alta direção; (2) uso intensivo pelos gestores } \\
\text { operacionais; (3) predominância de desafios e debates } \\
\text { face a face; (4) foco nas incertezas estratégicas; e (5) uma } \\
\text { forma não invasiva que facilita e inspira o envolvimento. } \\
\text { Mensuração deve ser feita como um construto formativo } \\
\text { em vez de reflexivo. }\end{array}$ \\
\hline
\end{tabular}

Quadro 3 - Síntese dos Trabalhos Analisados

\section{CONSIDERAÇÕES FINAIS}

Após detalhada revisão dos trabalhos, identifica-se que, de forma geral, grande parte do modelo teórico de Simons foi validada, o que o habilita como um construto a ser estudado empiricamente em pesquisas brasileiras. Muito embora existam estudos com resultados divergentes, isso estimula que futuras pesquisas brasileiras possam ser feitas tentando verificar se os resultados seriam similares às pesquisas internacionais. A análise identificou também que as pesquisas acabaram não tendo continuidade, com cada pesquisador desenvolvendo um aspecto do modelo e incorporando outros construtos oriundos de outros campos.

Quanto às contribuições dos estudos para as pesquisas brasileiras, identifica-se que os trabalhos de Henri (2006), Widener (2007) e Bisbe et al. (2007) são os com maiores potenciais para gerar pesquisas em Contabilidade Gerencial no Brasil, pois os dois primeiros possuem resultados divergentes, enquanto que o último pode ser utilizado para validar os procedimentos metodológicos sugeridos pelos autores. A aplicação do Uso Interativo em Pequenas Empresas também pode ser estudada, uma vez que o universo dessas empresas é muito grande e oferece potencial para publicação consoante o estudo de Collier (2005).

Quanto ao método, no atual estágio, pesquisas do tipo levantamento podem ser feitas no Brasil para validar o modelo teórico Levers of Control, muito embora estudos de casos, único ou múltiplo, preferencialmente com uma visão longitudinal, possivelmente propiciariam maiores contribuições para acadêmicos e a comunidade empresarial.

Quanto à abrangência de temas que podem ser estudados conjuntamente, esta é bastante ampla, mas é recomendável que as pesquisas possam ter continuidade, nesse sentido, podem ser aceitas as sugestões de Henri (2006) de incluir a variável incertezas estratégicas e operacionalizar as Tensões Dinâmicas como construto; além de seguir as recomendações metodológicas de Bisbe et al. (2007) sobre o enquadramento do construto Uso Interativo como modelo multidimensional. Estudos sobre a presença de Tensões Dinâmicas, Uso Diagnóstico e Uso Interativo, e influências destes na Estratégia também podem contribuir para o avanço das pesquisas. Por outro lado, é recomendável estudar este modelo como parte de um framework mais amplo consoante Ferreira e Otley (2009), 
O que se depreende dos trabalhos estudados é que não é recomendável, no estágio atual da pesquisa, relacionar as dimensões Desenho e/ ou Uso dos artefatos de Contabilidade Gerencial de forma direta com o Desempenho, sem que se incluam construtos oriundos da Estratégia. Nesse sentido é recomendável combinar o modelo teórico com outros construtos e teorias como Visão Baseada em Recursos, Aprendizagem Organizacional, Cultura Organizacional, Inovação, Comportamento Disfuncional, dentre outras possibilidades. Essa combinação é talvez a maior contribuição que o modelo Levers of Control propicia para as pesquisas em Contabilidade Gerencial.

A análise gerada neste trabalho tem como limitações o período de tempo escolhido e também a seleção dos principais periódicos internacionais, porém, pelos recentes artigos publicados pós 2007 se identifica que o tema ainda continua sendo estudado internacionalmente.

\section{REFERÊNCIAS}

ABERNETHY, M. A.; BROWNELL, P. The role of budgets in organizations facing strategic change: an exploratory study. Accounting, Organizations and Society, 24 , p. 189-204, 1999.

ARGYRIS, C.; SCHÖN, D. A. Organizational learning II: theory, method, and practice. Addison-Wesley, 1996.

BARNEY, J . Gaining and Sustaining Competitive Advantage. $3^{\text {rd }}$. Edition. Pearson, 2007.

BISBE, J.; OTLEY, D. The effects of the interactive use of management control systems on product innovation. Accounting, Organizations and Society, 29, p. 709 - 737, 2004.

; BATISTA-FOGUET, J. M; CHENHALL, R. Defining management accounting constructs: A methodological note on the risks of conceptual misspecification. Accounting, Organizations and Society, 32, p. 789 - 820, 2007.

; MALAGUEÑO, R. The Choice of Interactive Control Systems under Different Innovation Management Modes. European Accounting Review, v. 18, 2, p. $371-405,2009$.

BRUINING, H.; BONNET, M.; MIKE WRIGHT, M. Management control systems and strategy change in buyouts. Management Accounting Research, 15, p. 155 - 177, 2004.

CADEZ, S. ; GUILDING, C. An exploratory investigation of an integrated contingency model of strategic management accounting. Accounting, Organizations and Society, 33, p. 836-863, 2008.

CHAPMAN, C. ; COOPER, D. J .; MILLER, P. B. Linking Accounting, Organizations and Institutions. Accounting, Organizations and Institutions: Essay in Honour of Anthony Hopwood. Edited by CHAPMAN, C.; COOPER, D. J .; MILLER, P.B. Oxford, 2009.

COLLIER, P. M. Entrepreneurial control and the construction of a relevant accounting. Management Accounting Research, 16, p. 321 - 339, 2005.

DIMAGGIO, P. J.; POWELL, W. W. The Iron Cage Revisited: Institutional Isomorphism And Collective Rationality In Organizational Fields. American Sociological Review, v. 48, 2, Apr., p. 147 - 160, 1983.

FERREIRA, A.; OTLEY, D. Exploring inter and intra-relationships between the design and use of management control systems. SSRN. 2006.

. The Design and use of performance management systems: An 
extended framework analysis. Management Accounting Research, 20, p. 263 - 282, 2009.

FREZATTI, F. O paradigma econômico na contabilidade gerencial: um estudo empírico sobre a associação entre taxas de retorno sobre o patrimônio líquido e diferentes perfis da contabilidade gerencial. Revista de Administração. v. 41, 1, p. 5 - 17, jan./ fev./ mar. 2006.

FROW, N.; MARGINSON,D.; OGDEN, S. Encouraging strategic behaviour while maintaining management control: Multi-functional project teams, budgets, and the negotiation of shared accountabilities in contemporary enterprises. Management Accounting Research, 16, p. 269 - 292, 2006.

HOPWOOD, A. An empirical study of the role of accounting data in performance evaluation. J ournal of Accounting Research, v. 10, p. 156-182, 1972.

HENRI, J.F. Management Control Systems and Strategy: a Resource-based Perspective. Accounting, Organizations and Society , 31, p. 529-558, 2006.

J ÖNSSON, S. The Study of Controller Agency. In: Accounting, Organizations and Institutions: Essay in Honour of Anthony Hopwood. Edited by CHAPMAN, C.; COOPER, D.J .; MILLER, P. B. Oxford, 2009.

KOBER, R.; NG, J .; PAUL, B. J . The interrelationship between management control mechanisms and strategy. Management Accounting Research, 18, p. 425 - 452, 2007.

MARGINSON, D. E. W. Management control systems and their effects on strategy formation at middle-management levels: evidence from a UK organization. Strategic Management J ournal, 23, 11; p. 10 - 19, Nov. 2002.

MERCHANT, K. A. The Effects of Financial Controls on Data Manipulation and Management Myopia. Accounting, Organizations and Society, v. 15, 4, p. 297 - 313, 1990.

; VAN DER STEDE, W.A. Management Control Systems: Performance Measurement, Evaluation and Incentives. NOME DA CIDADE DA PUBLICAÇÃO Pearson, 2007.

MINTZBERG, H.; LAMPEL, J. Reflecting on the strategy process. Sloan Management Review, p. 21 - 30, Spring, 1999.

NARANJ O-GIL, D.; HARTMANN, F. How Top Management Teams Use Management Accounting Systems to Implement Strategy. J ournal of Management Accounting Research. V. 18, p. $21-53,2006$.

Management accounting systems, top management team heterogeneity and strategic change. Accounting, Organizations and Society, v. 32, 7-8, Oct.Nov. 2007.

OSTERLOH, M.; FREY, B.S. Does pay for performance really motivate employees? In: NELLY, A. (Org.) Business Performance measurement: Theory and practice. Cambridge University Press, 2002.

SILVA, E. D. da; SILVA, W. V.; CORSO, J. M. D. La implementación de la estratégia y el control estratégico: um análisis integrado. Revista de Economia e Administração. IBMEC São Paulo: v. 6, n. 4, out - dez 2007.

SIMONS, R. The Role of Management Control Systems in Creative Competitive Advantage: New Perspectives. Accounting, Organizations and Society. v. 15, p. 127-143, 1990.

Strategic Orientation and Top Management Attention to Control Systems. Strategic Management J ournal, v. 12, p. 49 - 62, 1991.

Levers of Control. Harvard Business School Publishing, 1995. 
. Performance Measurement and Control Systems for Implementing Strategy. Prentice Hall: New J ersey, 2000.

SOUZA, M. A.; LISBOA, L. P.; ROCHA, W. Práticas de Contabilidade Gerencial Adotadas por Subsidiárias Brasileiras de Empresas Multinacionais. Revista de Contabilidade \& Finanças USP. São Paulo: Fipecafi, 32, p.40 - 57, mai./ago. 2003.

TANI, T. Interactive control in target cost management. Management Accounting Research, 6, p. 399-414, 1995.

TUOMELA, T-S. The interplay of different levers of control: A case study of introducing a new performance measurement system. Management Accounting Research, 16, p. 293-320, 2005.

VANDENBOSHC, B. An empirical analysis of the association between the use of executive support systems and perceived organizational competitiveness. Accounting, Organizations and Society, 24, p. 77-92, 1999.

VAN DE VEN, A.H.; ENGLEMAN, R;M. Central Problems in Managing Corporate Innovation and Entrepreneurship p.47-72. In: SHEPHERD, D. A; KATZ, J. A. (Orgs). Corporate Entrepreneurship. Elsevier, 2004.

VAN DER STEDE, W.A. The relationship between two consequences of budgetary controls: budgetary slack creation and managerial short-term orientation. Accounting, Organizations and Society, v. 25, p. 609-622, 2000.

WIDENER, S. K. An empirical analysis of the levers of control framework. Accounting, Organizations and Society, 32, p. 757-788, 2007.

\section{ENDEREÇO DOS AUTORES:}

Universidade Presbiteriana Mackenzie, Centro de Ciências Sociais e Aplicadas.

R. da Consolação 896 - Prédio Rev. Modesto Carvalhosa

Consolação

01302-907 - Sao Paulo, SP - Brasil

Faculdade de Economia, Administração e Contabilidade

Universidade de São Paulo

Avenida Professor Luciano Gualberto, 908

Cidade Universitária

05508-900 - Sao Paulo, SP - Brasil

Fundação Instituto Capixaba de Pesq. em Contabilidade, Economia e Finanças.

Av. Fernando Ferrari, 1358

Goiabeiras

29075-010 - Vitória, ES - Brasil 